\title{
Computed Tomography as an Objective Measurement Tool for Secondary Lymphedema Treated With Extracorporeal Shock Wave Therapy
}

\author{
So-Yeon Kim, MD, Hasuk Bae, MD, Hye Min Ji, MD
}

Departments of Rehabilitation Medicine, Ewha Womans University School of Medicine, Seoul, Korea

Two patients with stage three secondary lymphedema of the upper extremities underwent treatment for breast cancer, including surgery, chemotherapy, and radiotherapy. They were examined with computed tomography (CT) before and after extracorporeal shock wave therapy (ESWT). We used a manual tracing method using PiViewSTAR software to calculate the volume of the upper extremities. There was a decrease in the volume of the subcutaneous compartment measured by CT before and after ESWT. CT may be helpful in determining the treatment target area of ESWT and to monitor the effect of treatment by measuring the changes in volume before and after ESWT in patients with lymphedema. Therefore, CT may have good clinical potential for treatment and follow-up in the management of lymphedema.

Keywords Lymphedema, Subcutaneous tissue, High-energy shock waves

\section{INTRODUCTION}

If the lymph drainage system is damaged by surgery or radiation therapy in the treatment of breast cancer, secondary lymphedema of the upper extremity can occur [1]. According to the classification of lymphedema by the World Health Organization Expert Committee on Fila-

Received June 18, 2014; Accepted August 29, 2014

Corresponding author: Hasuk Bae

Department of Rehabilitation Medicine, Ewha Womans University School of Medicine, 1071 Anyangcheon-ro, Yangcheon-gu, Seoul 158-710, Korea Tel: +82-2-2650-6020, Fax: +82-2-2650-5175, E-mail: acebhs@gmail.com

(c) This is an open-access article distributed under the terms of the Creative Commons Attribution Non-Commercial License (http://creativecommons. org/licenses/by-nc/4.0) which permits unrestricted noncommercial use, distribution, and reproduction in any medium, provided the original work is properly cited.

Copyright () 2015 by Korean Academy of Rehabilitation Medicine riasis, patients develop tissue with a spongy consistency because of increased fibrosis, which is considered to be 'non-pitting. In stage 2 lymphedema, the limb begins to harden and to increase in size. Irreversible swelling with hard, fibrotic, unresponsive tissue is characteristic of stage 3 lymphedema.

The effects of a combination of physical therapy and compression therapy for reducing lymphedema are limited in stages 2 and 3 because of dermatofibrotic changes [2]. Therefore, there is a need for treatment strategies for lymphedema that target the fibrotic tissues. Extracorporeal shock wave therapy (ESWT) has recently been studied as an alternative non-invasive therapy that induces therapeutic lymphangiogenesis by up-regulating the vascular endothelial growth factor and basic fibroblast growth factor [3-5]. In addition, ESWT has been found 
to be effective in improving skin elasticity, skin wellness, and promoting the organization of skin structures in patients with progressive systemic sclerosis [6].

However, because there is no appropriate tool to examine the changes in fibrotic tissues in patients with lymphedema, it is difficult to determine the therapeutic effects of ESWT [5]. The water displacement method, the circumferential volumetric method, and measurement methods using perometry are mainly used to clinically measure the volume of lymphedema. However, these methods have some disadvantages including difficulties in obtaining measurements, low accuracy, and the need for special devices [7]. In addition, palpation, the presence of Stemmer sign, and tonometry are used to measure the elasticity of the tissues. However, these methods are indirect and also have disadvantages due to low interand intra-rater reliability.

Until now, there is no consensus on a standard outcome measurement in lymphedema. In particular, there is no tool to evaluate both the volume of edema and the changes in the properties of the tissues in order to determine the therapeutic effects of ESWT. Computed tomography (CT) can accurately and easily measure the volume of edema using a cross-sectional area of the region of interest (ROI) in order to observe changes in the tissue properties. Therefore, CT may be suitable for determining the therapeutic effects of ESWT.

In this study, the authors performed CT scans in order to determine the type of therapy in patients with secondary lymphedema and fibrosis in the subcutaneous tissues and skins. Specifically, we determined whether ESWT should be applied and determined the region for intensive treatment. We report two cases where we used CT to assess the changes in the volume of the subcutaneous fat layers and the structure of the tissues before and after treatment.

\section{CASE REPORTS}

\section{Case 1}

A 50-year-old female patient reported to the Department of Physical Medicine and Rehabilitation of Ewha Womans University Mokdong Hospital as an outpatient with lymphedema in the right upper extremity. The patient had undergone breast-conserving surgery, axillary lymph node dissection, chemotherapy, and radiation therapy for breast cancer on the right side. After these treatments, secondary lymphedema occurred, and bandage therapy was administered as symptoms improved. However, edema from the forearm to the hand had been worsening for the previous month. The circumference of both arms was measured $5 \mathrm{~cm}$ above and below the elbow crease connecting the medial and lateral epicondyles. Compared to the left arm, the right arm had larger circumferences of $3.5 \mathrm{~cm}$ and $4.7 \mathrm{~cm}$ at $5 \mathrm{~cm}$ above and below the elbow, respectively. In addition, Stemmer sign and peau d'orange changes were observed. The range of motion of the right shoulder was limited. Flexion, abduction, and external rotation were $170^{\circ}, 150^{\circ}$, and $80^{\circ}$, respectively. According to these results, the patient had stage 3 lymphedema.

A CT scan of the upper extremities was performed using SOMATOM Sensation 16 (Siemens, Forchheim, Germany) in order to determine the location and volume of lymphedema and to establish a treatment plan. The patient underwent a CT scan with a slice thickness of 10 $\mathrm{mm}$ from the aortic arch to the tip of the fingers in the supine position with the shoulder at $180^{\circ}$ flexion, elbow extended, forearm pronated, and fingers extended. According to the results of the CT scan, the right medial forearm showed the most severe honeycomb pattern. When the right and left sides were compared, it was found that the thickness of the skin and subcutaneous fat had increased on the right side (Fig. 1A). The patient had severe skin hardening and underwent ESWT around the right forearm, and bandage therapy was continued. The ESWT was performed with Dornier AR2 (Dornier MedTech, Wessling, Germany), which is the electromagnetic defocused type. During one session of treatment, stimulation was applied 2,000 times. Stimulation was applied 1,000 times to the right medial forearm, which showed the most severe honeycomb pattern, and to the rest of the right upper limb. ESWT was performed twice a week for eight weeks, and a total of 16 sessions were administered. The intensity of treatment was $0.040-0.069 \mathrm{~mJ} / \mathrm{mm}^{2}$ energy flux density and the frequency was $5 \mathrm{~Hz}$.

After 16 sessions of ESWT, a CT scan of the upper extremity was performed to determine the therapeutic effects (Fig. 1B). The volume of the upper extremity was measured using a manual tracing technique with PiViewSTAR software (INFINITT Co. Ltd., Seoul, Korea) in order to examine the changes in volume in the areas with edema 

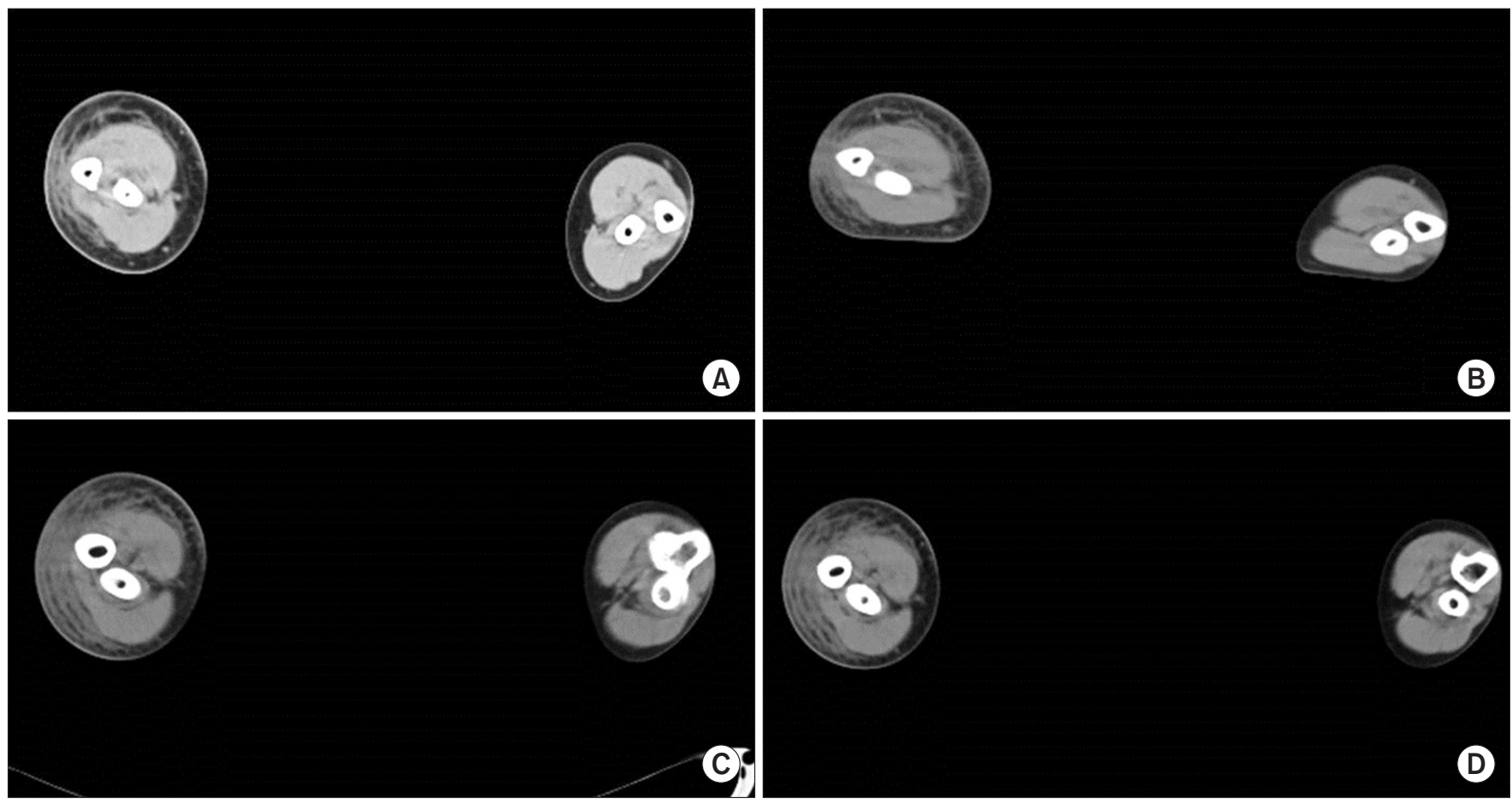

Fig. 1. Comparison of computed tomography of the upper extremity. Before (A) and after (B) treatment in the case 1 patient. Before (C) and after (D) treatment of the case 2 patient.

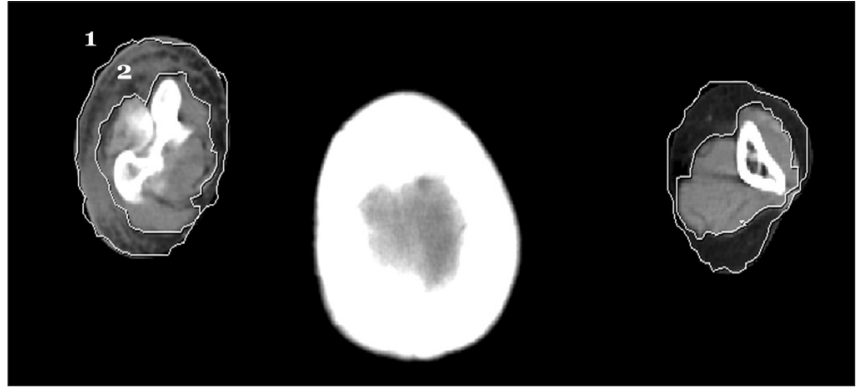

Fig. 2. Computed tomography based on the volume measurement method for lymphedema of the upper extremity whole extremity (1), the sum of bone and muscle (2).

(Fig. 2). The cross-sectional area observed in each section of the coronal CT scan was multiplied by the slice thickness (10 mm in this study) of the CT scan to obtain the volume of each section. The volume in the ROI was measured by adding up the volume of each section in the ROI. The volume of the subcutaneous fat layers was obtained by subtracting the volume of the bones and muscles from the volume of the entire upper extremity. The volume of the entire upper extremity was measured by summing up the volumes of the regions from the distal radial and ulnar styloid process to the acromion process. The volume of the areas between $5 \mathrm{~cm}$ below and $5 \mathrm{~cm}$ above the elbow was calculated and compared before and after treatment. The volume of the entire right upper extremity and the subcutaneous fat layers was reduced from $210,878.4 \mathrm{~mm}^{3}$ to $205,970.1 \mathrm{~mm}^{3}$ and from $122,562.2$ $\mathrm{mm}^{3}$ to $113,154.2 \mathrm{~mm}^{3}$, respectively. The volumes of the entire areas at $5 \mathrm{~cm}$ above the elbow and subcutaneous fat layers were reduced from $25,014.7 \mathrm{~mm}^{3}$ to $24,735.3$ $\mathrm{mm}^{3}$ and from $14,732.6 \mathrm{~mm}^{3}$ to $13,315.2 \mathrm{~mm}^{3}$, respectively. The volumes of the entire areas at $5 \mathrm{~cm}$ below the elbow and subcutaneous fat layers were reduced from $28,799.8 \mathrm{~mm}^{3}$ to $28,535.9 \mathrm{~mm}^{3}$ and from $15,243.2 \mathrm{~mm}^{3}$ to $13,935.6 \mathrm{~mm}^{3}$, respectively (Table 1 ). In addition, when the circumferences of both arms were compared, differences of $2.8 \mathrm{~cm}$ and $4.3 \mathrm{~cm}$ at $5 \mathrm{~cm}$ above and below the elbow, respectively, were noted. These circumference measurements were smaller than the initial measurements. However, the presence of Stemmer sign and peau d'orange changes persisted after therapy.

\section{Case 2}

A 53-year-old female patient with secondary lymph- 
Table 1. Volume of the upper extremity measured by computed tomography in the case 1 patient before and after $\operatorname{ESWT}\left(\mathrm{mm}^{3}\right)$

\begin{tabular}{|c|c|c|c|c|c|c|}
\hline \multirow{2}{*}{ Case 1} & \multicolumn{3}{|c|}{ Right } & \multicolumn{3}{|c|}{ Left } \\
\hline & Total & BE $5 \mathrm{~cm}$ & AE $5 \mathrm{~cm}$ & Total & BE $5 \mathrm{~cm}$ & AE $5 \mathrm{~cm}$ \\
\hline \multicolumn{7}{|l|}{ Pre ESWT } \\
\hline Whole & $210,878.4$ & $287,993.8$ & $25,014.7$ & $154,635.4$ & $18,917.6$ & $17,394.3$ \\
\hline Bone+muscle & $88,316.2$ & $13,556.7$ & $10,282.2$ & $91,014.8$ & $14,208.7$ & 9,933.5 \\
\hline Sub Q & $122,562.2$ & $15,243.2$ & $14,732.6$ & $63,620.6$ & $4,709.1$ & $7,460.9$ \\
\hline \multicolumn{7}{|l|}{ Post ESWT } \\
\hline Whole & $205,970.1$ & $28,535.9$ & $24,735.3$ & $154,930.1$ & $18,119.2$ & $17,474.6$ \\
\hline Bone+muscle & $92,815.9$ & $14,600.4$ & $11,420.1$ & $89,006.9$ & $13,944.6$ & $10,249.2$ \\
\hline Sub Q & $113,154.2$ & $13,935.6$ & $13,315.2$ & $65,923.1$ & $4,874.6$ & $7,225.4$ \\
\hline$\Delta$ Sub Q & $-9,408.1$ & $-1,307.6$ & $-1,417.4$ & $2,302.5$ & 165.5 & -235.5 \\
\hline Rate of change (\%) & -7.68 & -8.58 & -9.62 & 3.62 & 3.52 & -3.16 \\
\hline
\end{tabular}

AE $5 \mathrm{~cm}$, volume from elbow to $5 \mathrm{~cm}$ above the elbow; BE $5 \mathrm{~cm}$, volume from elbow to $5 \mathrm{~cm}$ below the elbow; ESWT, extracorporeal shock wave therapy; Sub Q, subcutaneous tissue; $\triangle$ Sub Q, difference before and after ESWT.

Table 2. Volume of the upper extremity measured by computed tomography in the case 2 patient before and after $\operatorname{ESWT}\left(\mathrm{mm}^{3}\right)$

\begin{tabular}{|c|c|c|c|c|c|c|}
\hline \multirow{2}{*}{ Case 2} & \multicolumn{3}{|c|}{ Right } & \multicolumn{3}{|c|}{ Left } \\
\hline & Total & BE $5 \mathrm{~cm}$ & AE $5 \mathrm{~cm}$ & Total & BE $5 \mathrm{~cm}$ & AE $5 \mathrm{~cm}$ \\
\hline \multicolumn{7}{|l|}{ Pre ESWT } \\
\hline Whole & $302,139.4$ & $37,066.3$ & $40,923.1$ & $189,492.1$ & $21,429.0$ & $23,541.5$ \\
\hline Bone+muscle & $114,420.0$ & $15,561.2$ & $14,826.3$ & $93,166.7$ & $12,007.6$ & $11,705.9$ \\
\hline Sub Q & $187,719.4$ & $21,505.2$ & $26,096.8$ & $96,325.4$ & $9,421.4$ & $11,835.6$ \\
\hline \multicolumn{7}{|l|}{ Post ESWT } \\
\hline Whole & $247,739.8$ & $29,701.3$ & $32,576.6$ & $180,053.3$ & $21,532.1$ & $24,188.4$ \\
\hline Bone+muscle & $100,555.2$ & $15,200.3$ & $12,693.7$ & $90,793.5$ & $13,086.4$ & $10,784.6$ \\
\hline Sub Q & $147,184.6$ & $14,500.9$ & $19,882.9$ & $89,259.7$ & $8,445.6$ & $13,403.8$ \\
\hline$\Delta$ Sub Q & $-40,534.9$ & $-7,004.2$ & $-6,213.9$ & $-7,065.7$ & -975.8 & $1,568.2$ \\
\hline Rate of change (\%) & -21.6 & -32.6 & -23.8 & -7.3 & -10.4 & 13 \\
\hline
\end{tabular}

AE $5 \mathrm{~cm}$, volume from elbow to $5 \mathrm{~cm}$ above the elbow; BE $5 \mathrm{~cm}$, volume from elbow to $5 \mathrm{~cm}$ below the elbow; ESWT, extracorporeal shock wave therapy; Sub Q, subcutaneous tissue; $\Delta$ Sub Q, difference before and after ESWT.

edema occurring after breast-conserving surgery, axillary lymph node dissection, chemotherapy, and radiation therapy for the treatment of breast cancer on the right side attended our outpatient lymphedema clinic. Her right arm had a larger circumstance than her left arm, with circumferences of $7.7 \mathrm{~cm}$ and $6.5 \mathrm{~cm}$ at $5 \mathrm{~cm}$ above and below the elbow, respectively. In addition, Stemmer sign was observed, but peau d'orange change was not. Thus, she was diagnosed with stage 3 lymphedema.

The patient underwent a CT scan of the upper extremity before she received treatment. A honeycomb pattern was observed in the right medial forearm and ventral side of the upper arm. The thickness of the skin and subcutaneous fat was increased on the right side (Fig. 1C). The patient underwent ESWT with the same method as performed in case 1.

When comparing the CT scans (Fig. 1D) performed before and after treatment, the volume of the entire right upper extremity and the subcutaneous fat layers was reduced from $302,139.4 \mathrm{~mm}^{3}$ to $247,739.8 \mathrm{~mm}^{3}$ and from $187,719.4 \mathrm{~mm}^{3}$ to $147,184.6 \mathrm{~mm}^{3}$, respectively. The volume of the entire area and the subcutaneous fat layers at 
$5 \mathrm{~cm}$ above the elbow was reduced from $40,923.1 \mathrm{~mm}^{3}$ to $32,576.6 \mathrm{~mm}^{3}$ and from $26,096.8 \mathrm{~mm}^{3}$ to $19,882.9 \mathrm{~mm}^{3}$, respectively. The volume of the entire area and the subcutaneous fat layers at $5 \mathrm{~cm}$ below the elbow was reduced from $37,066.3 \mathrm{~mm}^{3}$ to $29,701.3 \mathrm{~mm}^{3}$ and from $21,505.2$ $\mathrm{mm}^{3}$ to $14,500.9 \mathrm{~mm}^{3}$, respectively (Table 2). In addition, when the circumferences of both arms were compared, the difference was $5.0 \mathrm{~cm}$ and $4.8 \mathrm{~cm}$ at $5 \mathrm{~cm}$ above and below the elbow, respectively. These measurements were smaller than the initial measurements. However, the Stemmer sign observed in the right medial forearm persisted after treatment.

\section{DISCUSSION}

The two cases presented here differ from previous studies because a CT scan was performed to accurately measure volume and to examine the structural changes in patients with lymphedema. A CT scan of the upper extremity was helpful to detect areas with the most severe honeycomb pattern by continuously scanning the crosssections of the entire upper extremity after intensive treatment. As the volume of the entire area or of specific areas in the subcutaneous tissues and muscles can be selectively calculated, additional information was provided.

It is important to dynamically evaluate lymphedema in order to determine the type, areas involved, and the duration of treatment. In particular, accurate measurements of the size of the limbs are useful to obtain a basic understanding of the pathophysiology of lymphedema. The relationship between the volume of the upper extremity and the concentration of proteins deposited in the interstitial fluid, as well the relationship between the skin area and capillary density, showed a positive correlation in the edema occurring after mastectomy [8]. In the present case, a CT scan was used for selective volume measurements.

The major purpose of using CT scans to assess edematous limbs was to exclude the differential diagnosis of lymphedema due to other diseases, such as deep vein thrombosis, chronic venous congestion, lipoedema, hematoma, or popliteal cysts, by determining characteristics of the tissue and the degree of edema with a specific honeycomb pattern [7]. The honeycomb pattern found in patients with lymphedema is probably the result of an increase in the density of the interstitial fluid associated with chronic inflammation caused by collagen deposition due to the influx of macrophages and fibroblasts, interlobular septal hypertrophy, and fibrosis [9]. Therefore, the authors in this study targeted the areas that exhibited a concentrated honeycomb pattern in the upper extremity CT scan in patients with lymphedema.

Compared to magnetic resonance imaging (MRI) and ultrasonography, CT has some advantages in the assessment of lymphedema including its short examination time and high cost-efficiency. As MRI offers no significant differences in the assessment of nerve damage, axillary edema, and fibrosis compared to CT scans, it is of limited use given considerations of cost-efficiency and the convenience of examination. On the other hand, ultrasonography offers some advantages, such as accurate measurements of the thickness of skin and subcutaneous fat, but it has difficulty assessing an entire upper extremity with lymphedema [7]. Thus, ultrasonography is thought to be useful for assessing the progression of fibrosis after determining the areas of progressing fibrosis by comparing CT scans.

Although the effects of ESWT on patients with lymphedema are not clear, it has been found to have a cellular effect on lymphangiogenesis in animal models. Its benefits may be associated with cavitation and shear stress applied to the subcutaneous fat layers or with its mechanical effect $[3,4]$. Clinical trials assessing the effects of ESWT in patients with secondary lymphedema found reduced volumes of lymphedema and skin thickness and improved subjective evaluations of the hardness of the skin. It was also found that the progression of fibrosis was inhibited after ESWT in areas of fibrosis progression or initiation in patients with stage 3 lymphedema [5]. In the present cases, edema was reduced after ESWT, although there are some issues to consider when determining the treatment effect. Firstly, it was difficult to determine whether the effects were solely due to ESWT because the patient in case 1 continued to undergo bandage therapy. However, that patient used bandage therapy before she visited the hospital with worsening edema, which was significantly improved after she underwent a combination of bandage therapy and ESWT. Thus, it can be inferred that ESWT is effective in reducing edema. Secondly, it cannot be confirmed that tissue elasticity is improved in areas of edema. After initial evaluations with 
CT scans, ESWT was intensively applied to areas with the most severe 'honeycomb' pattern. However, when the CT scans that were performed before and after the treatment were compared, a reduction of volume was confirmed, but no reduction of the 'honeycomb' pattern was observed. It is believed that the density of the 'honeycomb' pattern may change as the volume of the upper extremity decreases. Therefore, in addition to CT scans, there is need for a tool that can evaluate tissue elasticity and the progression of fibrosis.

In conclusion, CT may be helpful in establishing the target treatment area of ESWT and for monitoring the effects of treatment by measuring the volume changes before and after ESWT in patients with secondary lymphedema with progressing fibrosis. CT may have good clinical potential for treatment and follow-up in the management of lymphedema.

\section{CONFLICT OF INTEREST}

No potential conflict of interest relevant to this article was reported.

\section{REFERENCES}

1. Bell RJ, Robinson PJ, Barallon R, Fradkin P, Schwarz M, Davis SR. Lymphedema: experience of a cohort of women with breast cancer followed for four years after diagnosis in Victoria, Australia. Support Care Cancer 2013;21:2017-24.

2. Korpan MI, Crevenna R, Fialka-Moser V. Lymphedema: a therapeutic approach in the treatment and rehabilitation of cancer patients. Am J Phys Med Rehabil 2011;90(5 Suppl 1):S69-75.

3. Serizawa F, Ito K, Matsubara M, Sato A, Shimokawa H, Satomi S. Extracorporeal shock wave therapy induces therapeutic lymphangiogenesis in a rat model of secondary lymphoedema. Eur J Vasc Endovasc Surg 2011;42:254-60.

4. Kubo M, Li TS, Kamota T, Ohshima M, Shirasawa B, Hamano K. Extracorporeal shockwave therapy ameliorates secondary lymphedema by promoting lymphangiogenesis. J Vasc Surg 2010;52:429-34.

5. Bae H, Kim HJ. Clinical outcomes of extracorporeal shock wave therapy in patients with secondary lymphedema: a pilot study. Ann Rehabil Med 2013;37:22934.

6. Tinazzi E, Amelio E, Marangoni E, Guerra C, Puccetti A, Codella OM, et al. Effects of shock wave therapy in the skin of patients with progressive systemic sclerosis: a pilot study. Rheumatol Int 2011;31:651-6.

7. Stanton AV, Badger C, Sitzia J. Non-invasive assessment of the lymphedematous limb. Lymphology 2000;33:122-35.

8. Roberts CC, Stanton AW, Pullen J, Bull RH, Levick JR, Mortimer PS. Skin microvascular architecture and perfusion studied in human postmastectomy oedema by intravital video-capillaroscopy. Int J Microcirc Clin Exp 1994;14:327-34.

9. Monnin-Delhom ED, Gallix BP, Achard C, Bruel JM, Janbon C. High resolution unenhanced computed tomography in patients with swollen legs. Lymphology 2002;35:121-8. 